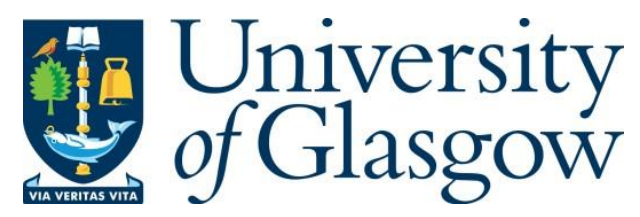

Xiao, Y., Zhang, L. and Imran, M. (2019) Active Constellation Extension for Peak

Power Reduction Based on Positive and Negative Iterations in OFDM Systems. In: 4th International Conference on UK - China Emerging Technologies (UCET 2019),

Glasgow, UK, 21-22 Aug 2019, ISBN 9781728127972.

There may be differences between this version and the published version. You are advised to consult the publisher's version if you wish to cite from it.

http://eprints.gla.ac.uk/190587/

Deposited on: 21 April 2020

Enlighten - Research publications by members of the University of Glasgow http://eprints.gla.ac.uk 


\section{Active Constellation Extension for Peak Power Reduction Based on Positive and Negative Iterations in OFDM Systems}

\author{
Yong Xiao \\ Department of satellite navigation \\ Center on Communication \& Navigation \\ Qinhuai District, Nanjing, China \\ Email: 18782418051@139.com
}

\author{
Lei Zhang \\ School of Engineering \\ University of Glasgow \\ Glasgow, United Kingdom \\ Lei.Zhang@glasgow.ac.uk
}

\author{
Muhammad Imran \\ School of Engineering \\ University of Glasgow \\ Glasgow, United Kingdom \\ Muhammad.Imran@glasgow.ac.uk
}

\begin{abstract}
Traditional active constellation extension (ACE) techniques iterate under a further and further away from decision boundary constraint to find distortions for peak-to-average power ratio (PAPR) reduction, which may stop the solution on suboptimal points because it's not permitted to go back when running into a suboptimum direction. In this paper, we present a novel ACE technique by iterating in both positive and negative directions, referring to distortions found in the last iteration. During iterations, optimization variations are changed from normally used extra distortions on the last estimates to the primitive OFDM signal, which can eliminate correlations between magnitudes and phases of complex distortions and finally give an analytic solution based on orthogonal projection. By making iterations run in positive and negative directions, this algorithm can find distortions to reduce PAPR more, compared with existing methods. Simulation results show that significant improvement can be achieved either for pure ACE or TR assisted ACE method, especially under higher-order modulation schemes.
\end{abstract}

Index Terms-OFDM, PAPR reduction, symbol distortion, ACE, projection.

\section{INTRODUCTION}

By modulating independent symbols on a series of parallel subcarriers, orthogonal frequency division multiplexing (OFDM) technology is inherently robust against frequency-selective fading channels, making it widely standardized in terrestrial communication systems such as WIFI [1], LTE [2] and 5G [3,4]. However, this modulation also causes the high peak-toaverage power ratio (PAPR) problem which obstacles the applications of OFDM under energy or layout constrained environments such as on satellite.

To mitigate this inborn high PAPR drawback, many approaches have been proposed [5, 6]. But except active constellation extension (ACE) [7], almost all of them may degrade bit error rate (BER) performance or need to occupy some extra subcarriers or become hard to implement when subcarrier number is huge, such as clipping and filtering $[8,9]$, tone reservation (TR) [10, $11]$, selective mapping $[12,13]$ and tone injection [14, 15]. By exploiting the distance character of modulation constellation, ACE can reduce PAPR effectively without changing the receiver or side information transmission [7]. Based on original ideas, a series of improvements have been proposed for ACE from perspectives such as clipping function choosing [16], subcarrier grouping optimization [17], etc. A bounded distortion method [18] had also been proposed to combine TR and ACE algorithms together. However, these existing methods only iterate in positive directions to promise no BER degradation. Any subcarrier distortion will be abandoned if it is not farther away from the decision boundary, compared with the last iteration. But the no BER degradation constraint only demands the total distortion on the primitive OFDM signal in far from decision boundary directions, not between all neighbor iterations. The over strict constraint adopted in existing methods always makes iterations stop on suboptimum points.

This paper presents iterations of ACE in a more general way. Expected distortions after each clipping are integrated with estimated distortions in the last iteration, by which to change the distortion basis from the last extended OFDM signal to the primitive. And then, these integrations are projected to valid zones subjected by far away from decision boundary constraint. Those projections, near to distortion integrations most, are adopted as final distortions to ACE subcarriers of this iteration. Compared with existing approaches to optimize extra distortion on the last iteration, this algorithm looks for optimized distortion integrated on the primitive OFDM signal by projection, which can provide opportunities to go backward when reaching suboptimum points, caused by amplitudes growing on former low samples and abandons of invalid distortions.

The rest of the paper is organized as follows. Section II presents fundamental definitions and concepts relating to the PAPR problem of OFDM, and basic ideas of ACE. In Section III, specifications of the proposed algorithm 
named as PB-ACE is derived. Section IV is devoted to performance comparisons for pure and TR assisted ACE under kinds of modulations including QPSK, 16-QAM and 64-QAM. Finally, our conclusions are given in Section V.

\section{PRELIMINARIES}

OFDM is a way to modulate a block of information on a series of parallel orthogonal subcarriers, which is represented as $X=\left\{X_{k}, k=0,1, \ldots, N-1\right\}$, supposing subcarrier number to be $N$. Its discrete baseband form in time-domain can be expressed as

$$
x_{n}=\frac{1}{\sqrt{N}} \sum_{k=0}^{N-1} X_{k} e^{j 2 \pi \frac{k n}{J N}}, n=0, \ldots, J N-1,
$$

where $J$ is the oversampling factor. From this specific equation, the peak power of this signal may be much higher than the average power when $N$ is large, according to the central limit theorem [19].

The ACE method is a kind of distortion technique by adding distortions on modulated subcarriers to reduce the peak power of primitive OFDM symbols. Signal after distortion can be represented as

$$
\hat{x}_{n}=x_{n}+d_{n}=\frac{1}{\sqrt{N}} \sum_{k=0}^{N-1}\left(X_{k}+D_{k}\right) e^{j 2 \pi \frac{k n}{J N}},
$$

where $d_{n}$ and $D_{k}$ respectively denote distortions on the $n$th sample and the $k$ th subcarrier. In this paper, small and capital letters are adopted to represent time-domain and frequency-domain variables respectively.

To measure power fluctuation degree, the PAPR may be defined as

$$
\varepsilon=\frac{\max _{n \in[0, J N-1]}\left|\hat{x}_{n}\right|^{2}}{E\left\{\left|X_{k}\right|^{2}\right\}} .
$$

The denominator of this fraction is not the distorted OFDM signal but the primitive, by which to avoid reference quantity variation caused by power increasing of the signal after ACE.

Because the average power of the primitive OFDM symbol during iteration is a constant, the reduction of PAPR can be simplified as a reduction of the maximum power, subjected to no BER degradation constraint. The formulation of PAPR reduction is specified as

$$
\underset{\mathbf{D}}{\operatorname{minimize}}\|\mathbf{x}+\mathbf{f D}\|_{\infty}^{2}
$$

S. T. $R e<D_{k}, X_{k}-X_{k}^{\prime}>\geq 0$ for all $k=\underset{X_{k}^{\prime} \in}{0, \ldots, N-1 \text { and }},(4 . \mathrm{b})$

where $\mathbf{x}=\left[x_{0}, \ldots, x_{J N-1}\right]^{T}, \mathbf{D}=\left[D_{0}, \ldots, D_{N-1}\right]^{T}$, and $\mathbf{f}$ means IFFT operator. $X_{k}^{\prime}$ represents any possible value in modulation constellation set $C$. $R e<,>$ means to get real part of the inner-product between two complex numbers [6].

For the $k$ th subcarrier, the constraint of (4.b) demands all real part of complex inner-products to be positive, between the distortion and vectors from any possible $X_{k}^{\prime}$ to $X_{k}$. This can be interpreted in a geometry way by mapping a complex to a point in a 2-D coordinate system. One positive inner-product constraint decides a valid region as half of a 2-D plane originated from $X_{k}$. The intersection of all these valid regions is the feasible region for this modulated data $X_{k}$. As all these half 2-D planes are originated from the same point, the intersection is either empty or an area rotated from a ray, which can be defined as a set specified as

$$
D=\left\{D_{k} \mid \operatorname{abs}\left(D_{k}\right) \leq b, \theta_{1} \leq \operatorname{phase}\left(D_{k}\right) \leq \theta_{2}\right\}
$$

$a b s()$ and phase() represent operators to get the amplitude and phase of a complex. The value of $b, \theta_{1}$ and $\theta_{2}$ are all depended on the modulation constellation and the specific data $X_{k}$ modulated on this subcarrier.

By extending modulation data in an intelligent way, time-domain peaks in the transmitted OFDM signal may be suppressed effectively. In reality, the optimization of (4) is always implemented in an iterative way to enhance search precision. By changing the basis from the original OFDM signal $\mathbf{x}$ to the distorted signal after $i-1$ th iteration $\hat{\mathbf{x}}^{\mathbf{i}-1}=\left[\hat{x}_{0}^{i-1}, \ldots, \hat{x}_{J N-1}^{i-1}\right]^{T}$, equations in (4) will be transformed into

$$
\underset{\overline{\mathbf{D}}^{\mathbf{i}}}{\operatorname{minimize}} \| \hat{\mathbf{x}}^{\mathbf{i}-\mathbf{1}}+\left.\mathbf{f} \overline{\mathbf{D}}^{\mathbf{i}}\right|_{\infty} ^{2}
$$

$$
\begin{array}{r}
\text { S. T. } R e<\bar{D}_{k}^{i}+\hat{X}_{k}^{i-1}-X_{k}, X_{k}-X_{k}^{\prime}>\geq 0 \text { for all } k= \\
0, \ldots, N-1 \text { and } X_{k}^{\prime} \in C .(6 . \mathrm{b})
\end{array}
$$

$\overline{\mathbf{D}}^{\mathbf{i}}=\left[\bar{D}_{0}^{i}, \ldots, \bar{D}_{N-1}^{i}\right]^{T}$ represents the expected distortion vector on the basis of the $i-1$ th distorted signal vector $\widehat{\mathbf{X}}^{\mathbf{i}-\mathbf{1}}$, while $\overline{\mathbf{D}}^{\mathbf{i}}+\widehat{\mathbf{X}}^{\mathbf{i}-\mathbf{1}}-\mathbf{X}$ means expected distortions on the basis of the primitive OFDM signal.

To make implementation easier, the constraint of (6.b) is approximated as $[7,16]$

$$
\begin{array}{r}
\text { S. T. } R e<\bar{D}_{k}^{i}, X_{k}-X_{k}^{\prime}>\geq 0 \text { for all } k=0, \ldots, N-1, \\
\text { and } X_{k}^{\prime} \in C,(7)
\end{array}
$$

which asks any distortion on the basis of the last iteration to be far away from decision boundary. Equation (7) is just a sufficient but not necessary condition of (6.b) because the inner-product between $\hat{X}_{k}^{i-1}-X_{k}$ and $X_{k}-$ $X_{k}^{\prime}$ is always positive, which shrinks the feasible region of (6), resulting in a suboptimal estimation.

\section{ACE BASED ON PROJECTION ITERATING IN POSITIVE AND NEGATIVE DIRECTIONS}

\section{A. Optimization formulation transformation}

Considering the constraint of (6.b), $\bar{D}_{k}^{i}$ may be valid even in a negative direction, as long as amplitude is small 
enough. Fig. 1 has shown this case under 16-QAM. Black squares and dots represent original points of this modulation, which have different extension regions identified as shaded half limited squares or lines. The red heave square identifies the complex value of one subcarrier before an iteration. After this iteration, the value is assumed to be distorted to the point identified as a red dotted square, which is nearer to decision boundary compared with the result of the last iteration. But the red dotted square is also valid because it's still in the black shaded region of this subcarrier.

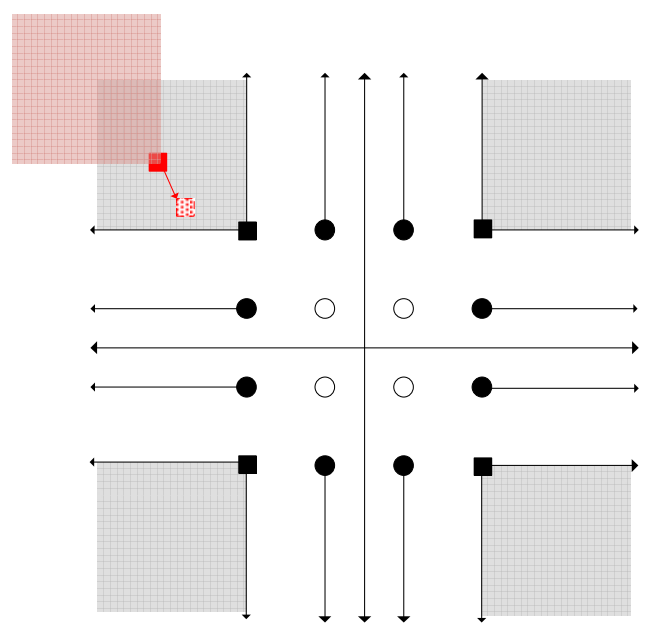

Fig. 1. An example of valid negative distortion during iteration.

The optimization of a complex variation as $\bar{D}_{k}^{i}$ can be interpreted as a joint optimization problem of two real variations as amplitude and phase. As the distorted data $\hat{X}_{k}^{i-1}$ is not equal to the primitive data $X_{k}$, the extension of the amplitude is limited in some directions constrained by (6.b). This makes amplitude and phase optimizations of $\bar{D}_{k}^{i}$ to be correlated, which is always difficult to implement. To eliminate the correlation between amplitude and phase during optimization, we adopt a new optimization variable $\overline{I_{-}} \bar{D}_{k}^{i}$ shown as

$$
\overline{I \_}_{k}^{i}=\bar{D}_{k}^{i}+{\widehat{I} D_{k}^{i-1}}^{i-1} \bar{D}_{k}^{i}+\hat{X}_{k}^{i-1}-X_{k},
$$

by which to transform the optimization formulation from (6) to

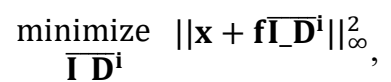

S. T. $R e<\bar{I}_{-} \bar{D}_{k}^{i}, X_{k}-X_{k}^{\prime}>\geq 0$ for all $k=0, \ldots, N-1$ and $X_{k}^{\prime} \in C,(9 . \mathrm{b})$

where $\overline{\mathbf{I}_{-} \mathbf{D}^{\mathbf{i}}}=\left[{\bar{I} D_{0}}_{0}^{i}, \ldots,{\bar{I} D_{N-1}^{i}}^{T}\right.$. In each iteration, the feasible region of $\bar{I}_{-} D_{k}^{i}$ is in a shape defined as (5). There is no correlation between phase and amplitude limitation, which makes the optimization of $\bar{I}_{-} \bar{D}_{k}^{i}$ easy to implement.

\section{B. Optimization formulation implementation}

In accordance with existing papers, optimization objective as (9) is implemented in a clipping way [20]. Expected clipping result in the $i$ th iteration is specified as

$$
\bar{x}_{n}^{i}= \begin{cases}A e^{j \varphi_{n}^{i-1},} & \left|\hat{x}_{n}^{i-1}\right|>A \\ \hat{x}_{n}^{i-1}, & \left|\hat{x}_{n}^{i-1}\right| \leq A\end{cases}
$$

$A$ is the expected maximum amplitude. $\hat{x}_{n}^{i-1}$ is the $n$th distorted sample after $i-1$ th iteration. Any sample higher than $A$ will be reduced to $A$ with phase invariant. This signal clipping is equal to add $\hat{x}_{n}^{i-1}$ with a time-domain distortion $\bar{d}_{n}^{i}$ specified as

$$
\bar{d}_{n}^{i}=\left\{\begin{array}{ll}
\left(A-\left|\hat{x}_{n}^{i-1}\right|\right) e^{j \varphi_{n}^{i-1}}, & \left|\hat{x}_{n}^{i-1}\right|>A \\
0, & \left|\hat{x}_{n}^{i-1}\right| \leq A
\end{array} .\right.
$$

Threshold $A$ can also be changed intelligently during iteration. As the focus of this paper is not clipping threshold adaptation, $A$ is simply set as a predefined constant.

Expected distortion on each subcarrier $\bar{D}_{k}^{i}$ can be easily achieved by DFT on time-domain distortion vector $\overline{\mathbf{d}}^{\mathbf{i}}=\left[\bar{d}_{0}^{i}, \ldots, \bar{d}_{J N-1}^{i}\right]$, which is shown as

$$
\bar{D}_{k}^{i}=\frac{1}{\sqrt{N}} \sum_{n=0}^{J N-1} \bar{d}_{n}^{i} e^{-j 2 \pi \frac{k n}{J N}} .
$$

Based on (8) and (12), we can get expected distortions to primitive OFDM signal shown as $\overline{\mathbf{I}} \overline{\mathbf{D}}^{\mathbf{i}}$. Some elements may satisfy the constraint of (9.b), which do not need a trim. But for other elements violating (9.b), the trim process is needed because of BER constraints.

The trim of ${\overline{\mathbf{I}} \mathbf{D}^{\mathbf{i}}}^{\mathbf{i}}$ is based on least square approximation (LSA) in this paper. The square error led by the trim in time-domain is specified in the first row of

$$
\begin{aligned}
E & =\sum_{n=0}^{J N-1}\left|l \bar{d}_{n}^{i}+\hat{x}_{n}^{i-1}-x_{n}-\mathbf{f}_{\mathbf{n}} \widehat{\mathbf{I}}_{-} \mathbf{D}^{\mathbf{i}}\right|^{2} \\
& =\sum_{k=0}^{N-1}\left|\mathbf{f}_{\mathbf{k}}^{-\mathbf{1}}\left(l \overline{\mathbf{d}}^{\mathbf{i}}+\hat{\mathbf{x}}^{\mathbf{i}-\mathbf{1}}-\mathbf{x}\right)-\widehat{I}_{-}^{i}\right|^{2} \\
& =\sum_{k=0}^{N-1}\left|{\bar{I} D_{k}^{i}}_{-}-{\widehat{I} D_{k}^{i}}^{i}\right|^{2} .
\end{aligned}
$$

$\mathbf{f}_{\mathbf{n}}$ and $\mathbf{f}_{\mathbf{k}}{ }^{-1}$ represent IDFT and DFT for the $n$th sample and the $k$ th subcarrier. Based on the orthogonal character between transform vectors, we can easily get a frequency-domain representation shown in the third row of (13).

Because feasible solutions for each subcarrier subjected to (9.b) is uncorrelated, the minimization of $E$ for all subcarriers is equal to minimize on each subcarrier independently. For a subcarrier whose extension region shown as (5) is empty, the value of $\widehat{I}_{-} D_{k}^{i}$ is kept as zero. For an extendable subcarrier, the magnitude $\hat{\rho}$ and phase $\hat{\theta}$ of $\widehat{I}_{-} D_{k}^{i}$ can be represented as 


$$
\begin{aligned}
& \underset{\theta_{1} \leq \hat{\theta} \leq \theta_{2}}{\operatorname{minimize}}\left\{\begin{array}{c}
\operatorname{minimize} \\
0 \leq \hat{\rho}<+\infty
\end{array} \mid \overline{I_{-} D_{k}^{i}-\left.\hat{\rho} e^{j \widehat{\theta}}\right|^{2}}\right\}= \\
& \operatorname{minimize}\left\{\left|\bar{I}_{-}^{i}{ }_{k}^{i}-\max \left(\operatorname{Re}<{\bar{I} D_{k}}_{k}^{i}, e^{j \widehat{\theta}}>, 0\right) e^{j \widehat{\theta}}\right|^{2}\right\} \\
& \theta_{1} \leq \hat{\theta} \leq \theta_{2}
\end{aligned}
$$

$\max ()$ means to get the bigger one between two real numbers. Based on projection theorem [21], the optimized ${\widehat{I_{D}}}_{D_{k}}^{i}$ can be deduced as

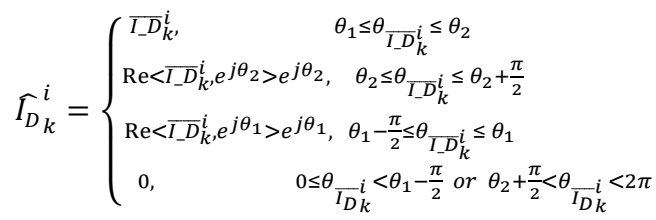

where $\theta_{\bar{I}_{-} \bar{D}_{k}^{i}}$ represents the phase of the complex $\bar{I}_{-} \bar{D}_{k}^{i}$.

\section{Complexity}

The whole algorithm can be implemented in a feedback mode concluded in the following TABLE. Because clipping and trim operations only involve in some simple scalar calculations on each sample or subcarrier, which are much smaller than those of DFT and IDFT operations involving complex vector innerproduct calculation [7]. The main computation complex of PB-ACE is dependent on DFT and IDFT in each iteration. In reality, as $J N$ time-domain samples are only based on $N$ independent subcarriers, there are some simple ways to decrease the computational complexity of DFT and IDFT on this kind of interpolated signal. And also, because all expected time-domain distortions are zeros on samples lower than $A$, the computational complexity of DFT is lower than common $J N$ length vector. The actual computation complex will lower than $O\left(2 J N \log 2^{J N}\right)$, which is the complexity for one standard DFT and IDFT of $J N$ length.

\begin{tabular}{l} 
PB-ACE Algorithm \\
\hline Initialization:
\end{tabular}

Load $(X, D)$ depended on specific information for transmission and modulation constellation.

Set $i=0, \widehat{\mathbf{I}_{-}} \mathbf{D}^{\mathbf{0}}=[0, \ldots, 0]^{T}$.

Select the clipping threshold $A$.

\section{Runtime:}

1: For $i=1,2, \ldots, I$

2: Calculate the time-domain signal vector $\mathbf{x}^{\mathrm{i}}$ based on the sum of $\mathbf{X}$ and $\widehat{\mathbf{I}}_{\mathbf{D}}^{\mathbf{i}-\mathbf{1}}$ as (2).

3: Clip $\mathbf{x}^{\mathbf{i}}$ to get time-domain distortion vector $\overline{\mathbf{d}}^{\mathbf{i}}$ based on (11) and calculate $\overline{\mathbf{D}}^{\mathbf{i}}$ by DFT as (12).

4: Add $\overline{\mathbf{D}}^{\mathbf{i}}$ on the final distortion vector of the last iteration to get expected distortion vector $\overline{\mathbf{I}_{-} \mathbf{D}^{\mathbf{i}}}$ of this iteration according to (8).

5: Trim expected distortion vector $\overline{\bar{I}_{-} \mathbf{D}^{\mathbf{i}}}$ based on
(15) to get the optimized vector $\widehat{\mathbf{C D}_{-}}$.

6: Until reach predefined iteration times $I$, output the distorted signal $\mathbf{x}^{\mathbf{I}}$ in the last iteration.

\section{SiMULATION RESULTS}

Different PAPR reduction techniques make different tradeoffs. Comparisons between them would be involved since it's hard to compare in a unified cost. Thus, we just simulate PB-ACE to compare with ACE series algorithms as ACE-SGP in [7], which is believed to be the most relevant algorithm for our comparisons. TR assisted ACE applications named as PB-ACE-TR and ACE-SGP-TR are also simulated to make comparisons.

During simulations, the OFDM signal is a complex baseband signal with 256 subcarriers using $10^{6}$ randomly generated symbols. Oversampling factor and iteration times are all set to 8. Modulation modes include QPSK, 16-QAM and 64-QAM. The goal of PAPR reduction is to cut down all larger than $6 \mathrm{~dB}$ cases which would higher than $24 \mathrm{~dB}$ in the worst. All simulations are constrained by no BER degradation promise. Performances are compared in terms of CCDF [7].

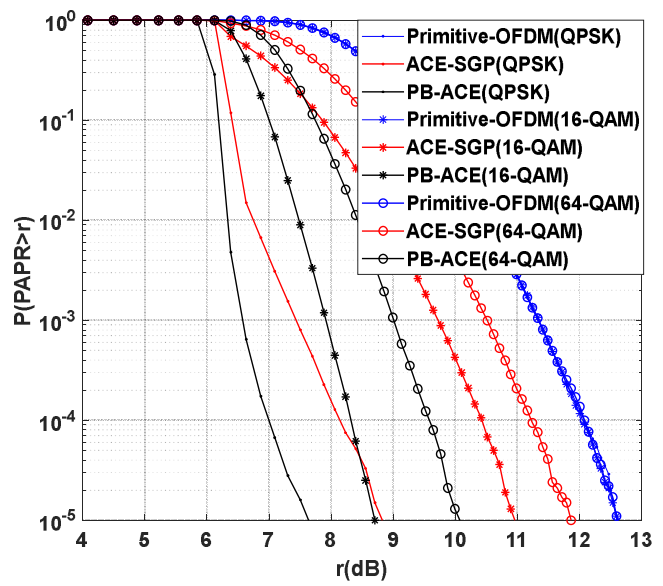

Fig. 2. PAPR comparison for pure ACE under different modulations.

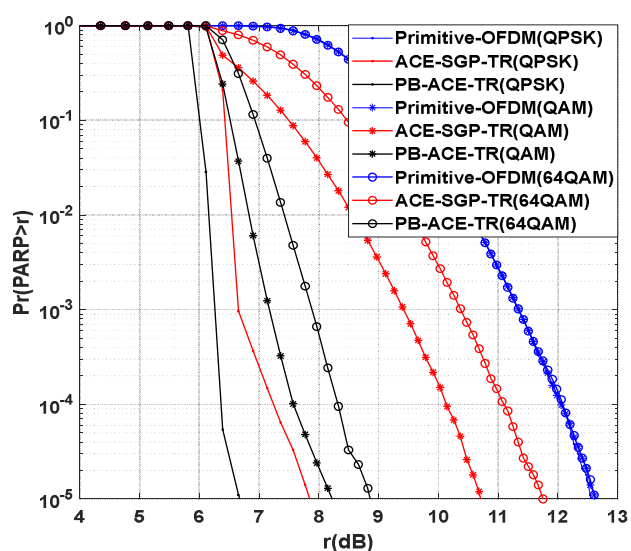

Fig. 3. PAPR comparison for TR assisted ACE under different modulations. 
Fig. 2 and Fig. 3 have shown simulation results for pure ACE and TR assisted ACE respectively. PrimitiveOFDM represents the case without any PAPR reduction distortion. Compared with existing ACE-SGP algorithm, pure ACE application of PB-ACE can gain a $1 \mathrm{~dB}$ PAPR improvement at the probability of $10^{-4}$, while near $2 \mathrm{~dB}$ under 16-QAM or 64-QAM modulation. When combined with TR, the PAPR improvement is near $3 \mathrm{~dB}$ under 64-QAM modulation. It looks that existing ACESGP becomes more and more inefficient when the modulation order becomes higher or combined with TR, compared with the algorithm proposed in this paper.

\section{CONCLUSION}

A novel ACE algorithm iterating in both positive and negative directions has been presented. The algorithm transforms the iterative optimization variation from extra distortion on the last iteration to distortion on primitive OFDM signal, which eliminate correlations between amplitude and phase of a complex distortion on any subcarrier. With the uncorrelated amplitude and phase representation, an analytic solution based on LSA can be achieved by projection, which is much easier to implement compared with convex optimization ways. By iterating in both positive and negative directions, the presented algorithm shows a clear performance improvement compared with ACE-SGP, especially considering higher-order modulations or combined with TR algorithm.

\section{REFERENCES}

[1] R. B. M. Abdelrahman, A. B. A. Mustafa and A. A. Osman, "A Comparison between IEEE 802.11a, b, g, n and ac Standards," IOSR-JCE, vol. 17, iss. 5, pp. 26-29, Oct. 2015.

[2] Z. Shen, A. Papaskellariou, J. Montojo, D. Gerstenberger and F. Xu, "Overview of 3GPP LTE-Advanced Carrier Aggregation for 4G Wireless Communications," IEEE Commun. Mag., vol. 50, iss. 2, pp. 122-130, Feb. 2012.

[3] A. Ijaz et al., "Enabling Massive IoT in 5G and Beyond Systems: PHY Radio Frame Design Considerations," in IEEE Access, vol. 4, pp. 3322-3339, 2016.

[4] X. Zhang, L. Zhang, P. Xiao, D. Ma, J. Wei and Y. Xin, "Mixed Numerologies Interference Analysis and InterNumerology Interference Cancellation for Windowed OFDM Systems," IEEE Transactions on Vehicular Technology, vol. 67, no. 8, pp. 7047-7061, Aug. 2018.

[5] Y. Rahmatallah and S. Mohan, "Peak-To-Average Power Ratio Reduction in OFDM Systems: a Survey and Taxonomy," IEEE Commun. Surv. Tutor., vol. 15, no. 4, Fourth Quarter 2013.

[6] Dr. V. Nandalal and G. Gelvakumar, "Power Optimization in OFDM Networks Using Various Peak to Average Power Ratio Techniques," Asian Journal of
Applied Science and Technology (AJAST), vol. 1, iss. 2, pp. 185-199, Mar 2017.

[7] B. S. Krongold and D. L. Jones, "PAPR reduction in OFDM via active constellation extension," IEEE Trans. Broadcast., vol. 49, pp. 258-268, Sept. 2003.

[8] J. Armstrong, "Peak-to-average power reduction for OFDM by repeated clipping and frequency domain filtering," Electron. Lett., vol. 38, no. 5, pp. 246-247, Feb. 2002.

[9] Y. C. Wang and Z. Q. Luo, "Optimized iterative clipping and filtering for PAPR reduction of OFDM signals," IEEE Trans. Commun., vol. 59, no. 1, pp. 33-37, January 2011.

[10] H. Li, T. Jiang and Y. Zhou, "An Improved Tone Reservation Scheme with Fast Convergence for PAPR Reduction in OFDM Systems," IEEE Trans. Broadcasting., vol. 57, no. 4, pp. 902-906, Dec. 2011.

[11] Y. Wang and Z. Luo, "Optimized Iterative Clipping and Filtering for PAPR Reduction of OFDM Signals," IEEE Trans. Commun., vol. 59, no. 1, pp. 33-37, Jan 2011.

[12] S. H. Muller and J. B. Huber, "A comparison of peak power reduction schemes for OFDM," in Proc. IEEE Global Communications Conference(GLOBECOM), Phoenix, AZ, November 1997, pp. 1-5.

[13] R. W. Bauml, R. F. H. Fisher, and J. B. Huber, "Reducing the peak to average power ratio of multicarrier modulation by selected mapping," IEEE Electronic Letters, vol. 32, no. 22, pp. 2056-2057, October 1996.

[14] C. Hwang, "A peak power reduction method for multicarrier transmission," in Proc. IEEE International Conference on Communications(ICC), vol. 5, June 2001, pp. 1496-1500.

[15] S. H. Han, J. M. Cioffi, and J. H. Lee, "Tone injection with hexagonal constellation for peak-to-average power ratio reduction in OFDM," IEEE Commun. Lett., vol. 10, no. 9, pp. 646-648, September 2006.

[16] C. Li, T. Jiang, Y. Zhou, and H. Li, "A novel constellation reshaping method for PAPR reduction of OFDM signals," IEEE Trans. Signal Process., vol. 59, no. 6, pp. 27102719, Jun. 2011

[17] S. Wang, W. Lin, B. Huang and C. Li, "PAPR Reduction in OFDM Systems Using Active Constellation Extension and Subcarrier Grouping Techniques," IEEE Commun. Lett., vol. 20, no. 12, pp. 2378-2381, Dec. 2016.

[18] S. K. Deng and M. C. Lin, "Recursive clipping and filtering with bounded distortion for PAPR reduction," IEEE Trans. Commun., vol. 55, no. 1, pp. 227-230, January 2007.

[19] John G.Proakis, Digital Communications. McGraw-Hill, 2007.

[20] A. Gatherer and M. Polley, "Controlling clipping probability in DMT transmission," in Proc. 31 st Asilomar Conference on Signals, Systems, and Computers, 1997, pp. 578-584.

[21] Xianda Zhang, Matrix Analysis and Applications. Tsinghua University Press, 2004. 